Proceedings of the American Control Conference Philadelphia, Pennsylvania • June 1998

\title{
Optimization of Network Signal Timing
}

\author{
Xiao-Hua Yu, Allen R. Stubberud \\ xhyu@ece.uci.edu, arstubbe@uci.edu \\ Department of Electrical and Computer Engineering \\ University of California, Irvine \\ Irvine, CA 92697
}

\begin{abstract}
A typical urban traffic network is a complicated large-scale stochastic system which consists of many interconnected signalized traffic intersections. This paper develops a decentralized real-time adaptive control strategy for the traffic networks based on Markov decision theory. Computer simulation results of this new approach on a five intersection traffic network indicate significant improvement over the traditional fully actuated control algorithm.
\end{abstract}

\section{INTRODUCTION}

Setting signal timing at intersections so that the traffic in a region containing many intersections flows efficiently is a key goal in traffic management of urban networks. The conventional urban traffic control (UTC) systems operate on the fixed timing plans which are generated off-line based on the deterministic traffic conditions during different time periods of the day (e.g., peak hours, off-peak hours), and thus do not respond to the fluctuations of the traffic flows in the network. TRANSYT-7F (TRAFFIC NETWORK STUDY TOOL) [1] is a well-known and effective software package to implement this methodology. SCOOT (SPLIT, CyCle and OfFSET OPtimization TeChNiQue) [2] and SCATS (SydNEY COORDINATED ADAPTIVE TRAFFIC SYSTEM) [3] are generally considered to be "on-line" algorithms, in which the control strategy is to "match" the current traffic conditions obtained from detectors to the "best" precalculated off-line timing plan. Some recent on-going researches apply new technologies to achieve real-time adaptive traffic signal control, such as artificial neural network [5], rule based expert system [6], fuzzy logic controller [7], etc.

The Markov decision process, or the controlled Markov process, has been studied by many researchers and has found applications in many areas. A discrete time, stationary Markov control model is defined on $(\mathrm{X}, \mathrm{A}, \mathrm{P}, \mathrm{R})$ where $\mathrm{X}$ is the state space and every element $\mathrm{x} \in \mathrm{X}$ is called a state; $\mathrm{A}$ is the set of all possible controls (or alternatives); $\mathrm{P}$ is a probability measure space, in which an element $p_{i, j}^{k}$ denotes the transition probability from state $i$ to state $j$ under alternative $\mathrm{k}$; and $\mathrm{R}$ is a measurable function, also called a one-step reward.

Choosing a particular alternative results in an immediate reward and a transition probability to the next step. The ultimate objective is to find the supremum (least upper bound) of the total expected discounted reward over an infinite period of time:

$$
J \triangleq E\left[\sum_{t=0}^{\infty} \beta^{t} r\left(x_{t}, a_{t}\right)\right]
$$

where $r$ is the one-step transition reward, $\beta(0 \leq \beta$ $<1)$ is the discount factor, and a is the policy. The optimal reward $\mathrm{v}^{*}$ is defined as:

$$
\mathrm{v}^{*}\left(\mathrm{x}, \mathrm{a}^{*}\right)=\sup _{\mathrm{a} \in \mathrm{A}}[\mathrm{J}(\mathrm{x}, \mathrm{a})]
$$

It can be obtained by solving a DPE (dynamic programming equation):

$$
\mathrm{v}^{*}=\mathrm{Tv}^{*},
$$

where $\mathrm{T}$ is a contraction mapping and:

$$
\operatorname{Tv}(x)=\max _{a \in A}\left[r(x, a)+\beta \sum_{j=1}^{N} v(x) p_{i, j}^{a}\right] .
$$

It has been proved that the optimal solution of the above DPE is unique and can be calculated iteratively by the successive approximation method [4]:

$$
v_{n}(x)=\max _{a \in A}\left[r(x, a)+\beta \sum_{j=1}^{N} v_{n-1}(x) p_{i, j}^{a}\right]
$$

In the following sections, a new approach based on the above Markov decision theory is proposed and applied to the traffic signal control problem. 


\section{THE DYNAMIC MODEL OF TRAFFIC NETWORK}

A small traffic network consisting of two four-legged intersections is shown in Fig. 1. In each individual intersection, there are eight movements and the numbers of movements are labeled according to NEMA (National Electrical Manufacturers Association) convention. These eight movements of each intersection can be further classified into two different types, i.e., external movement and internal movement. For example, movement 1 and 6 are internal movements of intersection 1 , which receive the outputs from intersection 2, movement 3 and 6. All the other movements of intersection 1 are external movements.

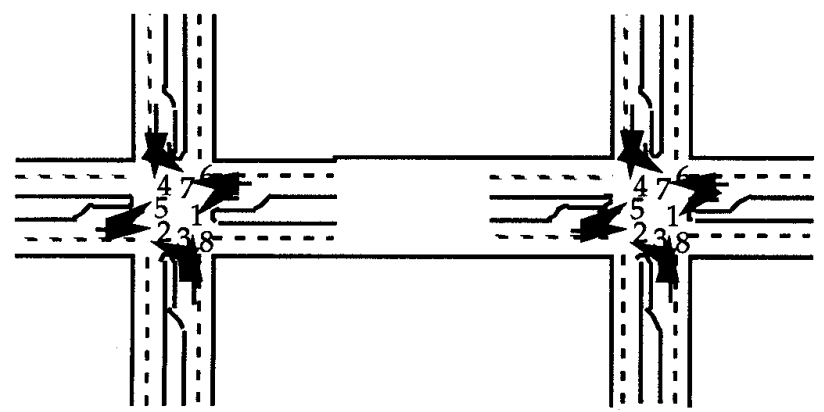

Intersection I

Intersection II

Figure 1. Two intersections

Consider a continuous traffic flow process which is sampled every $\Delta t$ time interval with the discrete time index, $\mathrm{k}$. The output of the network (i.e., number of vehicles leaving this network) $\underline{\mathrm{q}_{\text {out }}}(\mathrm{k})$ can be defined as a vector:

$\underline{\mathrm{q}_{\text {out }}}(\mathrm{k})=\left[\mathrm{q}_{\text {out } 1}{ }^{1}(k), \ldots, \mathrm{q}_{\text {out } 1}^{8}(\mathrm{k}), \mathrm{q}_{\text {out } 2}^{1}(\mathrm{k}), \ldots, \mathrm{q}_{\text {out } 1}^{8}(\mathrm{k})\right]^{\mathrm{T}}$

where the element of this vector $q_{\text {out } i}^{j}(k)$ denotes the output queue of the $\mathrm{j}$-th movement $(\mathrm{j}=1,2$, $\ldots, 8)$ of the $i$-th intersection $(i=1,2) . \quad q_{\text {out }}(k)$ can be further expressed as a function of the current control of the intersection, $\underline{u}(\mathrm{k})$, and the current queue $q(\mathrm{k})$ :

$$
\underline{\mathrm{q}_{\text {out }}}(\mathrm{k})=\underline{\mathrm{f}_{\text {out }}}(\underline{\mathrm{u}}(\mathrm{k}), \underline{\mathrm{q}}(\mathrm{k}))
$$

where $\underline{u}(k)$ and $\underline{f_{\text {out }}}(k)$ are also vectors and

$$
\mathrm{f}_{\text {out }_{\mathrm{i}}}^{\mathrm{j}}(\bullet)= \begin{cases}\min \left[\mathrm{q}_{\mathrm{i}}^{\mathrm{j}}(\mathrm{k}), \frac{\Delta \mathrm{t}}{\mathrm{h}_{\min }}\right] & \text { when } \mathrm{u}_{\mathrm{i}}^{\mathrm{j}}(\mathrm{k})=\text { Green } \\ 0 & \text { when } \mathrm{u}_{\mathrm{i}}^{\mathrm{j}}(\mathrm{k})=\operatorname{Red}\end{cases}
$$

where $\mathrm{i}=1,2$, and $\mathrm{j}=1,2, \ldots, 8$. $\mathrm{h}_{\min }$ is the minimum headway; $u_{i}^{j}(k)$ is the control signal for the $j$-th movement of the $i$-th intersection.

The current queue, $q(k)$, can be written as:

$$
\underline{\mathrm{q}}(\mathrm{k})=\underline{\mathrm{q}}(\mathrm{k}-1)+\underline{\mathrm{q}_{\text {in }}}(\mathrm{k})-\underline{\mathrm{q}_{\text {out }}}(\mathrm{k})
$$

where $q(k-1)$ is the queue at the previous time instant $(\mathrm{k}-1)$ and $\mathrm{q}_{\mathrm{in}}(\mathrm{k})$ is the input (number of vehicles) during time interval $[\mathrm{k}-1, \mathrm{k})$. Note that for the internal movement,

$$
\mathrm{q}_{\mathrm{in}_{\mathrm{i}}}^{\mathrm{j}}(\mathrm{k})=\mathrm{f}_{\mathrm{in}}^{\mathrm{j}}\left[\beta_{\mathrm{m}, \mathrm{i}}^{1, \mathrm{j}}\left(\mathrm{k}-\mathrm{T}_{\mathrm{m}, \mathrm{i}}^{1}\right), \mathrm{q}_{\text {out }_{\mathrm{m}}}^{1}\left(\mathrm{k}-\mathrm{T}_{\mathrm{m}, \mathrm{i}}^{1}\right)\right]
$$

where $q_{i n i}^{j}(k)$ is the input queue of the $j$-th movement of intersection $i$, and $q_{\text {out }}^{j}(k)$ is the output queue of $\mathrm{j}$-th movement of intersection $\mathrm{i}$. $\mathrm{T}$ is the minimum travel time between intersection 1 and 2:

$$
\mathrm{T}_{2,1}^{3}=\frac{\mathrm{L}_{2,1}^{3}}{\mathrm{v}_{2,1}^{3}}, \quad \mathrm{~T}_{2,1}^{6}=\frac{\mathrm{L}_{2,1}^{6}}{\mathrm{v}_{2,1}^{6}}
$$

where $\mathrm{L}_{2,1}^{3}=\mathrm{L}_{2,1}^{6}$ is the distance between intersections 2 and $1, v_{2,1}^{3}$ is the speed of the first vehicle of the platoon moving from intersection 2 , movement 3 to intersection 1 , etc. $\beta_{m, i}^{1, j}$ is defined as the vehicle turning factor from intersection $\mathrm{m}$, movement $l$ to intersection $i$, movement $j$. For this case, $\beta(k)$ can also be written as a matrix:

$$
\underline{\beta}(\mathrm{k})=\left[\begin{array}{c:c}
\beta_{1,1}(\mathrm{k}) & \beta_{1,2}(\mathrm{k}) \\
\hdashline \widehat{\beta}_{2,1}(\mathrm{k}) & \underline{\beta_{2,2}}(\mathrm{k})
\end{array}\right]
$$

where $\beta_{1,1}(k)$ represents the turning factor from intersection $i$ to intersection $\mathrm{j}(\mathrm{i}, \mathrm{j}=1,2)$.

The time duration of the current traffic signal, $\tau$, must be bounded between some minimum and maximum time period:

$$
\tau_{\min } \leq \tau \leq \tau_{\max }
$$

In an eight-phase dual-ring control, to avoid conflict traffic, only certain signal sequences are allowed. Since there are up to 3 admitted phases in each ring, when choosing current control, three previous control signals need to be 
considered in order to satisfy the sequence constraint:

$$
\underline{\mathrm{u}}(\mathrm{k})=\underline{\mathrm{f}} \underline{\mathrm{u}}\left(\underline{\mathrm{q}}(\mathrm{k}), \tau, \underline{\mathrm{u}}\left(\mathrm{k}-\tau_{1}\right), \underline{\mathrm{u}}\left(\mathrm{k}-\tau_{2}\right), \underline{\mathrm{u}}\left(\mathrm{k}-\tau_{3}\right)\right)
$$

\section{TRAFFIC SIGNAL CONTROL USING MARKOV DECISIONS}

To apply Markovian decision control to traffic systems, a state space $X$ and a probability measure $P$ must be defined. A threshold (number of vehicles) is chosen for the queue of each movement at an intersection. If the queue length of a specific movement is greater than the threshold value, then this movement is defined to be in its congestion mode; otherwise it is in the non-congestion mode. These two modes (congestion/non-congestion) are defined as the two states in the state space $X$. The signal phasing can be considered as different alternatives in each state. If there are eight independent movements for an intersection under eight-phase signal control, then the traffic control problem can be formatted as a 256-state Markov process with 8 alternatives in each state.

The probability matrix $\underline{P}(\mathrm{k})$ is a function of the current queue, the estimated number of arrivals in the next time interval, and the control signal. It can be further specified based on different arrival patterns. Studies on the occurrence of vehicle arrivals at traffic intersections found that: (1) the probability of the occurrence is related with the average arrival rate and the time interval, and do not vary in time; (2) two or more arrivals (of the same movement at the same lane) cannot occur at the same time; and (3) the chance of an occurrence in $(t, t+\Delta t]$ is independent of what happens before $t$. Supported by the results of many field tests, Kinzer (1933), Adams (1936) and Greenshilds (1947) et. al. claimed that under most circumstances, the arrival of vehicles for the external movements follows the Poisson distribution. Therefore,

$$
\mathrm{p}(\mathrm{n})=\frac{(\lambda \Delta \mathrm{t})^{\mathrm{n}} \mathrm{e}^{-\lambda \Delta \mathrm{t}}}{\mathrm{n} !}
$$

where $n=1,2, \ldots, \lambda$ is the arrival rate and $\Delta t$ is the time interval. Assuming that at a specific time instant, the current queue length of a specific movement $i$ is denoted by $q^{i}$, and there are $q_{g}^{i}$ vehicles passing through the intersection if the control signal of this direction is green, then:

$$
\dot{p}_{s_{i} \rightarrow N_{i}}^{u_{i}}=p\left(\hat{q}_{\text {in }}^{i}+q^{i}-\delta\left(u_{i}\right) q_{g}^{i} \leq q_{\text {threshold }}^{i}\right)
$$

and

where

$$
\mathrm{p}_{\mathrm{s}_{\mathrm{i}} \rightarrow \mathrm{C}_{\mathrm{i}}}^{\mathrm{u}_{\mathrm{i}}}=1-\mathrm{p}_{\mathrm{s}_{\mathrm{i}} \rightarrow \mathrm{N}_{\mathrm{i}}}^{\mathrm{u}_{\mathrm{i}}}
$$

$$
\delta\left(u_{i}\right)=\left\{\begin{array}{l}
1, \text { when } u_{i}=G_{i} \\
0, \text { otherwise }
\end{array}\right.
$$

and $S_{i}=N_{i}, C_{i}\left(N_{i}\right.$ for non-congestion and $C_{i}$ for congestion); $u_{i}=G_{i}, R_{i}\left(G_{i}\right.$ for green signal and $\mathrm{R}_{\mathrm{i}}$ for red signal).

For the internal movement, the probability can be calculated by Robertson's platoon dispersion formula (if a green signal is given at the upstream intersection; otherwise the probability is $0)$. For a reference point and a reference direction in a traffic network, an upstream intersection is defined as the intersection from which the traffic flow on the reference point comes; and the downstream intersection is defined as the intersection to where the traffic flow on the reference point goes.

Robertson's platoon dispersion is a very effective model to describe the inter-network traffic flow dynamics. It is based on the fact that the vehicles leaving from the upstream intersection are grouped into a "platoon" moving to the downstream intersection by the upstream green light:

$$
\begin{aligned}
& Q_{1}\left(t_{0}+T\right)=F \cdot Q_{2}\left(t_{0}\right)+(1-F) \cdot Q_{1}\left(t_{0}+T-1\right) \\
& \text { and } F=\frac{1}{1+\alpha \beta T_{\text {avg }}}
\end{aligned}
$$

where $Q_{1}, Q_{2}$ is the traffic volume at the downstream/upstream intersection (veh/hr), respectively; $\alpha$ and $\beta$ are called platoon dispersion parameters; $t_{0}$ is the initial time when the platoon leaves the upstream intersection; $\mathrm{T}_{\text {avg }}$ is the average travel time and $\mathrm{T}$ is minimum travel time between the two intersections $\left(T, T_{\text {avg }}\right.$ and $t_{0}$ are all rounded integers). The probability of a single vehicle arriving at the downstream intersection at time step $\mathrm{n}$ can be derived:

$$
\mathrm{p}(\mathrm{n})=(1-\mathrm{F})^{\mathrm{n}} \mathrm{F}
$$

The reward matrix $R$ has the same dimension and a similar definition to the probability matrix. The control objective herein is to minimize the queue length, so the functions of 
queue length corresponding to different states are chosen to be the elements of the reward matrix:

$$
\mathrm{R}_{\text {state } 1 \text {, state } 2}^{\mathrm{u}_{\mathrm{i}}}=\mathrm{f}_{\mathrm{u}}\left(\mathrm{q}_{0}^{\mathrm{i}}, \mathrm{q}_{\text {threshold }}^{\mathrm{i}}, \mathrm{u}_{\mathrm{i}}\right)
$$

Once the transition matrix and the reward matrix are obtained, a certain policy of choosing a certain alternative in each state, which is the optimal strategy we should take, will be obtained by maximizing the total expected reward. It has been shown that this optimal solution is unique and can be calculated iteratively [4]. Thus, the problem of choosing signal phasing becomes a decision-making problem for a Markov process.

In real-time traffic control problems, both the probability matrix and reward matrix are timevarying. In this paper, the sampling time is chosen the same as the minimum green extension time, $\Delta t$. Every $\Delta t$ seconds, the $P$ and $R$ matrices are calculated; then a decision is made to choose the control signal for the next time interval based on the current measurement from the detector and our estimation. Once the optimal policy is found, it is implemented only for $\Delta t$ seconds. At the next time step, the probability matrix and reward matrix are updated and the whole decision-making process is repeated.

\section{SIMULATION RESULTS}

A complicated traffic network can be decomposed into a group of small networks called "elementary networks". Fig. 2 shows such a small network consisting of five intersections.

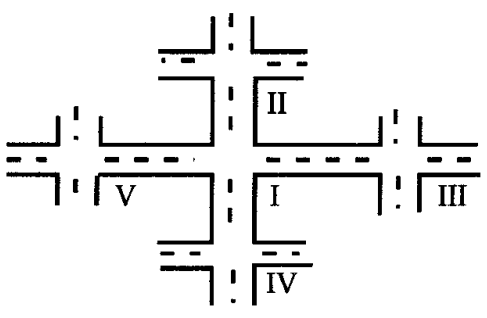

Fig. 2. A typical five intersection traffic network

The proposed Markovian adaptive control algorithm was simulated for this network (with the Poisson arrival pattern generated as the external inputs) to evaluate its performance in comparison to a fully actuated control method. The two algorithms were tested on four different arrival rates, i.e., $\lambda=200,300,400,500$ and 600 vehicles per hour per movement. For each arrival rate, the algorithms were tested on forty different sets of random data. The mean, covariance and standard deviation of the average steady state delay (of the 40 sets of data) were calculated and are listed in table 1, where "MAC" stands for the Markov adaptive control algorithm, and "FAC" stands for the fully actuated control. The means (of the 40 sets of data) of the steady state delay are also plotted in Fig. 3, where the solid line represents the Markov algorithm and the dotted line represents the fully actuated control.

Using the concept of distribution-free order statistics, the limits within which at least $90 \%$ of the probability of the steady state delay obtained from simulation are found in Table 2, with $92 \%$ confidence. In other words, the probability that $90 \%$ of the probability of the delay time lies between the lower and upper bound is 0.92 . These bounds are also plotted in Figure 4.

Table 1. Mean, covariance, and standard deviation of two algorithms

\begin{tabular}{|c|c|c|c|c|c|c|c|c|c|c|}
\hline & \multicolumn{2}{|c|}{200} & \multicolumn{2}{c|}{300} & \multicolumn{2}{c|}{400} & \multicolumn{2}{c|}{500} & \multicolumn{2}{c|}{600} \\
\cline { 2 - 12 } & FAC & MAC & FAC & MAC & FAC & MAC & FAC & MAC & FAC & MAC \\
\hline Mean & 23.10 & 12.46 & 28.64 & 12.96 & 35.21 & 14.91 & 43.25 & 20.44 & 62.71 & 52.33 \\
\hline Cov. & 2.29 & 0.95 & 4.46 & 2.09 & 4.34 & 2.24 & 4.60 & 3.96 & 9.13 & 38.63 \\
\hline Std. & 1.51 & 0.98 & 2.11 & 1.45 & 2.08 & 1.50 & 2.15 & 1.99 & 3.02 & 6.22 \\
\hline
\end{tabular}


Table 2. Bounds for simulation results

\begin{tabular}{|c|c|c|c|c|c|c|c|c|c|c|}
\hline & \multicolumn{2}{|c|}{200} & \multicolumn{2}{c|}{300} & \multicolumn{2}{c|}{400} & \multicolumn{2}{c|}{500} & \multicolumn{2}{c|}{600} \\
\cline { 2 - 11 } & FAC & MAC & FAC & MAC & FAC & MAC & FAC & MAC & FAC & MAC \\
\hline $\begin{array}{c}\text { Lower } \\
\text { limit }\end{array}$ & 20.0 & 10.57 & 25.0 & 10.68 & 30.3 & 12.45 & 38.7 & 14.99 & 55.7 & 41.98 \\
\hline $\begin{array}{c}\text { Upper } \\
\text { limit }\end{array}$ & 25.6 & 15.19 & 32.9 & 18.40 & 39.6 & 19.33 & 49.0 & 24.36 & 68.8 & 65.46 \\
\hline
\end{tabular}

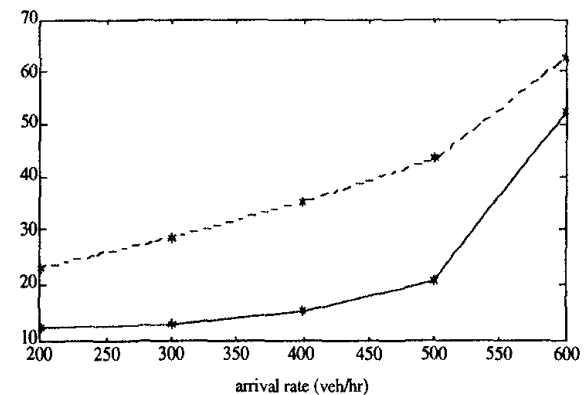

Figure 3. Mean of two algorithms

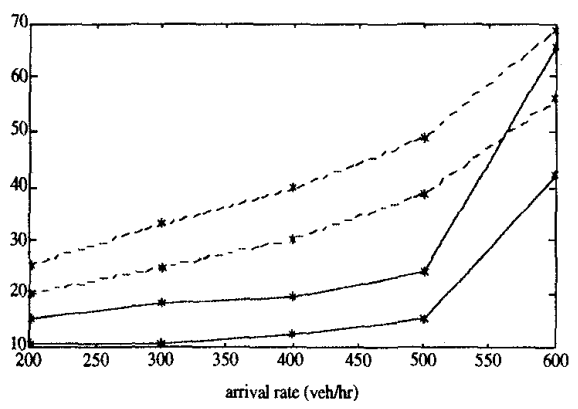

Figure 4. Bounds for simulation results

From the above data, it is shown that when the traffic intersection is under saturated, the Markov algorithm outperforms the traditional one. For example, when $\lambda \leq 500$, the average steady state delay of the Markov controller is only about one half of that of the fully actuated controller. However, when the intersection becomes saturated (e.g., $\lambda=600$ ), a large delay for both algorithms is found.

\section{CONCLUSIONS}

The traffic system is a stochastic system. In this paper, a new decentralized adaptive control scheme with embedded platoon dispersion is developed to minimize the queue length and the steady state delay of traffic networks. Computer simulation results and analysis on a typical network with five intersections are also presented.

\section{ACKNOWLEDGMENT}

This research work was supported by the California Department of Transportation, under its Advanced Transportation Management Systems Testbed Program.

\section{REFERENCES}

[1] Transportation research center, Traffic network study tool: TRANSYT-7F software summary, University of Florida, 1987

[2] Hunt, P. B., Robertson, D. I., et al., "The SCOOT on-line traffic signal optimization technique", Traffic engineering and control, April 1982

[3] Lowrie, P., "The Sydney coordinated adaptive control system -- Principles, methodology, algorithms", IEE conference publication, vol. 207, 1982

[4] Hernandez-Lerma, O., Adaptive Markov control process, Applied mathematical sciences 79, Springer-Verlag, 1989

[5] Nataksuji, T., Kaku, T., "Development of a self-organizing traffic control system using neural network models", Transportation Research Record 1324,1991

[6] Elahi, S. M., and Radman, A. E., Knowledge based system for adaptive traffic signal control, Transportation research record 1324, 1991

[7] McDonald, M., Wu, J., Brackstone, M., "Development of a fuzzy logic based microscopic motorway simulation model", IEEE International Conference on Intelligent Transportation Systems, 1997 